\title{
Molecular prevalence, characterization and associated risk factors of Anaplasma spp. and Theileria spp. in small ruminants in Northern Pakistan
}

\author{
Sadaf Niaz ${ }^{1}$, Zia Ur Rahman ${ }^{1}$, Ijaz Ali ${ }^{1}$, Raquel Cossío-Bayúgar ${ }^{2}$, Itzel Amaro-Estrada ${ }^{2}$, Abdullah D. Alanazi ${ }^{3}$, \\ Irfan Khattak ${ }^{1}$, Jehan Zeb ${ }^{1}$, Nasreen Nasreen ${ }^{1}$, and Adil Khan ${ }^{1, *}$ \\ ${ }^{1}$ Department of Zoology, Abdul Wali Khan University Mardan, Toru Road, Sheikh Maltoon Town, 23200 Mardan, Pakistan \\ ${ }^{2}$ Centro Nacional de Investigación Disciplinaria en Salud Animal e Inocuidad, Instituto Nacional de Investigaciones \\ Forestales Agrícolas y Pecuarias INIFAP, Carr. Fed. Cuernavaca-Cuautla No. 8534, Jiutepec, 62550 Morelos, México \\ ${ }^{3}$ Department of Biological Sciences, Faculty of Science and Humanities, Shaqra University, P.O. Box 1040, \\ 11911 Ad-Dawadimi, Saudi Arabia
}

Received 1 September 2020, Accepted 14 December 2020, Published online 8 January 2021

\begin{abstract}
This study was conducted in four districts (Malakand, Swat, Bajaur and Shangla) of Northern Pakistan to investigate the prevalence, associated risk factors and phylogenetic analyses of Theileria and Anaplasma species in small ruminants. A total of 800 blood samples, 200 from each district, were collected from apparently healthy animals. PCR assays were performed using generic primers for Anaplasma spp. and Theileria spp. as well as species specific primers for A. ovis and T. ovis. Overall infection prevalence was 361/800 (45.1\%). Theileria spp. infection prevalence (187/800, 23.3\%) was higher than Anaplasma spp. (174/800, 21.7\%). Amplified partial 18S rRNA genes were sequenced and enrolled animals were found to be infected by T. ovis (115/800,14.3\%), and at least two more Theileria species $(72 / 800,9 \%)$ were present (T. lestoquardi and T. annulata). All blood samples that were found to be positive for Anaplasma spp. were also positive for A. ovis. Infection prevalence was higher in sheep (227/361, 28.3\%) compared to goats $(134 / 361,16.6 \%)(p<0.005)$. Univariable analysis of risk factors showed that host, age, grazing system and acaricide treatment were significant determinants $(p<0.05)$ for both Theileria and Anaplasma infections. Multivariable analysis revealed that host, sex, age, tick infestation and grazing system were significant risk factors $(p<0.005)$ for both pathogens. Phylogenetic analysis revealed variants among the A. ovis and T. annulata samples analysed, indicating that different genotypes are circulating in the field while $T$. ovis presented the same genotype for the samples analysed.
\end{abstract}

Key words: Anaplasma ovis, Theileria annulata, Theileria ovis, Theileria lestoquardi, Sheep, Goat, Phylogeny, Risk factors, Pakistan.

\begin{abstract}
Résumé - Prévalence moléculaire, caractérisation et facteurs de risque associés d'Anaplasma spp. et Theileria spp. chez les petits ruminants du nord du Pakistan. Cette étude a été menée dans quatre districts (Malakand, Swat, Bajaur et Shangla) du nord du Pakistan pour étudier la prévalence, les facteurs de risque associés et les analyses phylogénétiques des espèces de Theileria et Anaplasma chez les petits ruminants. Au total, 800 échantillons de sang, 200 de chaque district, ont été prélevés sur des animaux apparemment sains. Les tests PCR ont été réalisés en utilisant des amorces génériques pour Anaplasma spp. et Theileria spp. ainsi que des amorces spécifiques à l'espèce pour $A$. ovis et $T$. ovis. La prévalence globale de l'infection était de 361/800 (45,1\%). La prévalence de l'infection à Theileria spp. (187/800, 23,3\%) était plus élevée que celle d'Anaplasma spp. (174/800, 21,7\%). Le gène de l'ARNr partiel $18 \mathrm{~S}$ amplifié a été séquencé et les animaux concernés se sont révélés infectés par $T$. ovis $(115 / 800,14,3 \%)$ et au moins deux autres espèces de Theileria $(72 / 800,9 \%)$ étaient présentes (T. lestoquardi et T. annulata). Tous les échantillons de sang trouvés positifs pour Anaplasma spp. ont également été trouvés positifs pour $A$. ovis. La prévalence de l'infection était plus élevée chez les moutons $(227 / 361,28,3 \%)$ que chez les chèvres $(134 / 361,16,6 \%)(p<0,005)$. Une analyse univariée des facteurs de risque a montré que l'hôte, l'âge, le système de pâturage et le traitement acaricide étaient des déterminants significatifs $(p<0,05)$ pour les infections à Theileria et Anaplasma. L'analyse multivariée des facteurs de risque a révélé que l'hôte, le sexe, l'âge, l'infestation par les tiques et le système de pâturage étaient des éléments de facteurs de risque importants $(p<0,005)$ pour les
\end{abstract}

*Corresponding author: zoologyawkum@gmail. com

This is an Open Access article distributed under the terms of the Creative Commons Attribution License (https://creativecommons.org/licenses/by/4.0), which permits unrestricted use, distribution, and reproduction in any medium, provided the original work is properly cited. 
deux agents pathogènes. L'analyse phylogénétique a révélé des variantes parmi les échantillons d'A. ovis et de $T$. annulata analysés indiquant que différents génotypes circulent sur le terrain tandis que $T$. ovis présentait le même génotype pour tous les échantillons analysés.

\section{Introduction}

Sheep and goats play a key role in the economy of Pakistan [66]. This is shown by the fact that Pakistan has the third largest goat population (76.1 million) and the 12th largest sheep population (30.9 million) in the world [27]. The Pakistani livestock sector contributed $11.2 \%$ to the gross domestic product (GDP) in 2018-2019, and a total of 48.8 thousand tons of meat and its products were exported during this period, generating income of US\$ 198.8 million and representing approximately $3.1 \%$ of total national exports. It is estimated that livestock production is the source of $35-40 \%$ of income for over 8 million rural families in Pakistan [41]. The Punjab and Sindh provinces of Pakistan have suitable land for crops and have more developed industrial sectors, but the population of Khyber Pakhtunkhwa (KP) province is highly dependent on livestock production and it significant contributes to livelihoods [13, 39]. Successful livestock production is hampered by a variety of factors such as mismanagement, infestation by ectoparasites, contagious infectious diseases, and nutritional deficiencies, preventing KP from fulfilling the meat, milk and hide requirements for an increasing human population [58]. One main cause of this inefficiency is tick-borne diseases [23]. The most important tick-borne diseases reported from tropical and sub-tropical areas are theileriosis and anaplasmosis, caused by intraerythrocytic pathogens of the genera Theileria and Anaplasma, respectively [19, 45]. There are six Theileria species that cause ovine theileriosis in small ruminants: Theileria (T.) ovis, T. separata, T. uilenbergi, T. recondita, T. lestoquardi, and $T$. luwenshuni $[34,47,52,61$, 63]. Among the Theileria spp., T. ovis, T. recondita and T. separata have low pathogenicity [8]. Theileria luwenshuni and T. uilenbergi are considered more pathogenic, while T. lestoquardi, causing malignant theileriosis, is the most pathogenic among all small ruminant Theileria species [51]. The clinical signs of theileriosis consist of fever, nasal discharge, jaundice, anaemia, lacrimation, enlargement of superficial lymph nodes, rapid weight loss, anorexia, reduced appetite and anaemia [39, 57]. Anaplasma species that are considered to infect small ruminants are A. marginale, A. ovis, and $A$. phagocytophilum, and a recently identified A. capra [46, 48]. Anaplasma capra is a zoonotic species detected in humans and sheep, and goats, cattle and wild ruminants are considered to be reservoirs [40]. Anaplasma phagocytophilum is the causative agent of granulocytic anaplasmosis, which infects the neutrophils of humans and causes tick-borne fever in small ruminants, dogs and horses [62]. Of these Anaplasma spp., A. marginale and A. ovis infect small as well as large ruminants worldwide and have considerable economic importance [30, 36]. Anaplasma ovis is moderately pathogenic in sheep, goats and wild ruminants [53]. Anaplasma marginale is related to acute anaplasmosis characterized by pale mucous, weight loss, severe anaemia, pyrexia, jaundice, decreased milk production, nervous signs, constipation, dehydration, loss of wool, coughing, laboured breathing, abortion, and sometimes death of the infected animal [1]. Ticks of Hyalomma spp. and
Haemaphysalis spp. are known to transmit Theileria spp. to small ruminants [9] while anaplasmosis is biologically transmitted to ruminants by about 20 species of ixodid ticks including Rhipicephalus spp., Hyalomma spp. and Dermacentor spp. Among these, Rhipicephalus microplus is globally reported to be the major Anaplasma spp. vector tick [30]. Mechanical transmission of Anaplasma spp. by the genera Tabanus, Stomoxys, and Psorophora or through contaminated fomites is frequent $[11,31,48]$.

Various risk factors such as the sex of the animal, herd management, seasons, tick presence, and herd size are reported to be associated with theileriosis and anaplasmosis. Spatio-temporal conditions such as vector habitat, bacterial populations, animal grazing systems, hygiene and management practices also affect the epidemiology of the infection [32]. The aim of this study was to estimate the prevalence of Theileria spp. and Anaplasma spp. in sheep and goat blood samples collected from four districts in northern Pakistan and to report the risk factors that are associated with the prevalence of these pathogens.

\section{Materials and methods \\ Ethics approval}

The study design and experimental protocols were approved by the ethics committee of the Department of Zoology, Abdul Wali Khan University Mardan, Pakistan (approval number Awkum-Z00-1125). Written and informed consent was obtained from veterinary hospitals, from where samples were collected, and from livestock owners.

\section{Study area and number of samples}

A total number of 800 blood samples were collected between January and December 2019 from four districts (Malakand $\left[34^{\circ} 30^{\prime} 10.8^{\prime \prime} \quad \mathrm{N}, \quad 71^{\circ} 54^{\prime} 16.56^{\prime \prime} \quad \mathrm{E}\right], \quad$ Swat $\left[35^{\circ} 13^{\prime} 21.72^{\prime \prime} \mathrm{N}, 72^{\circ} 25^{\prime} 32.88^{\prime \prime} \mathrm{E}\right]$, Bajaur [34 $34^{\circ} 47^{\prime} 11.4^{\prime \prime} \mathrm{N}$, $\left.71^{\circ} 32^{\prime} 5.64^{\prime \prime} \mathrm{E}\right]$ and Shangla [34 $\left.53^{\prime} 13.92^{\prime \prime} \mathrm{N}, 72^{\circ} 45^{\prime} 25.2^{\prime \prime} \mathrm{E}\right]$ ) of northern Pakistan (Fig. 1). A random sampling approach was applied to collect blood samples from symptomatic and asymptomatic goats $(n=401)$ and sheep $(n=399)$. From each district, 200 blood samples were collected, and enrolled animals included 281 males and 519 females. In these districts, livestock are managed on pastures as well as stall feeding under a traditional management system. These areas are sub-tropical dry mixed deciduous scrub forest and dry subtropical temperate semi-evergreen scrub forest. The annual average temperature of the studied areas ranges from 20 to $40{ }^{\circ} \mathrm{C}$ during the hottest months of the year with $65 \%$ average humidity [48].

\section{Demographic characteristic of the study population}

A total of 800 goats and sheep were studied, among which almost equal numbers of goats $401(50.1 \%)$ and sheep 399 


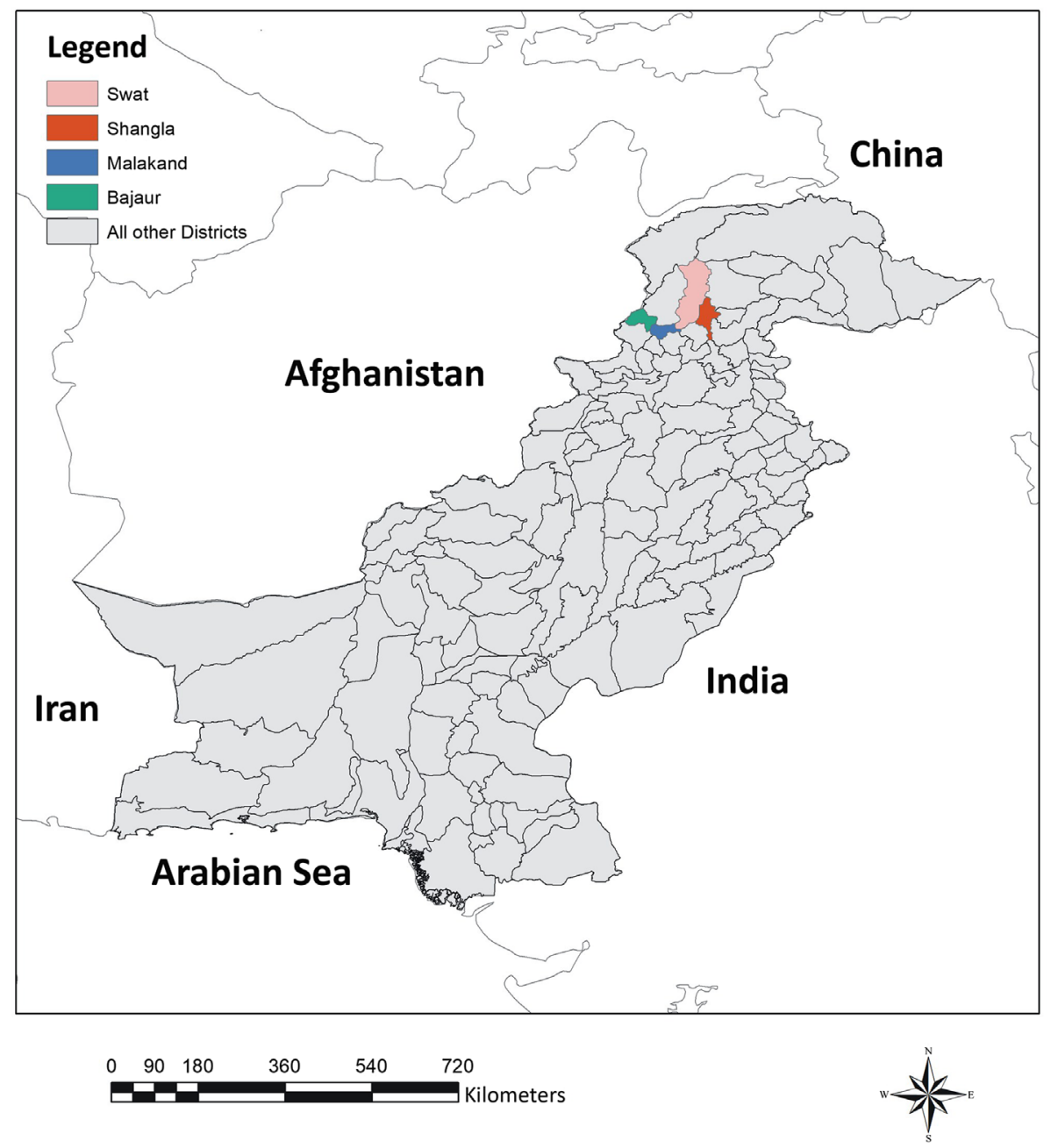

Figure 1. Map showing geographic location of study area (Northern Pakistan).

(49.9\%), with animals $<6$ months and animals $\geq 6$ months of age also included with the same ratio of $50.1 \%$ and $49.9 \%$, respectively. Sex-based sampling analysis indicated that the majority of the enrolled animals were female $(n=519$; $64.9 \%)$ goats and sheep as compared to males $(n=281$; $35.1 \%)$. The majority of the sampled animals were freegrazing $(n=300 ; 37.5 \%)$ followed by semi-grazing $(n=196$; $24.5 \%$ ) and zero-grazing animals (animals with stall feeders restricted to their homes) $(n=304 ; 38 \%)$. A total of $265(33.1 \%)$ goats and sheep were treated regularly, 200 $(25 \%)$ were irregularly treated with acaricides and 335 (41.9\%) never received any acaricidal treatment. About 452 $(65.5 \%)$ of the investigated population was not infected by ticks (Table 1).

\section{Risk factors analysis}

A questionnaire was designed to gather data regarding the risk factors (age, sex, host type (goat or sheep), acaricide applications, grazing system, tick infestation) associated with the studied pathogens, and it was completed on the spot at the time of blood sampling. Goats and sheep were categorised into two age groups: $<6$ months and $\geq 6$ months of age. The whole body of each animal was thoroughly inspected for tick infestations.

\section{Blood sample collection}

Blood samples were collected in EDTA tubes (Thermo Fischer Scientific ${ }^{\mathrm{TM}}$, USA) and kept at $+4{ }^{\circ} \mathrm{C}$ until they were further processed at the parasitology laboratory of the College of Veterinary Sciences and Animal Husbandry, Abdul Wali Khan University Mardan, Pakistan.

\section{Molecular detection of Theileria and Anaplasma species}

DNA was extracted from the preserved blood samples with a Gene all Kit (Exgene ${ }^{\mathrm{TM}}$ Blood SV, Germany), according to the manufacturer's instructions. DNA was quantified using a NanoDrop ${ }^{\mathrm{TM}} 1000$ spectrophotometer (NanoDrop Technologies Inc., Wilmington, DE, USA) based on 260/280 nm absorbance ratios, and then stored at $-80{ }^{\circ} \mathrm{C}$ for future analyses.

PCR-based assays were used to screen the collected samples. Generic primers that amplify the $16 \mathrm{~S}$ small subunit 
Table 1. Demographic properties of the study population from study area.

\begin{tabular}{|c|c|c|c|c|c|c|}
\hline Variables & Categories & Shangla & Swat & Malakand & Bajaur & Total $(\%)$ \\
\hline \multirow[t]{2}{*}{ Age } & Young & 70 & 74 & 69 & 71 & $401(50.1)$ \\
\hline & Adult & 130 & 126 & 131 & 129 & $399(49.9)$ \\
\hline \multirow[t]{2}{*}{ Sex } & Male & 75 & 67 & 64 & 75 & $281(35.1)$ \\
\hline & Female & 125 & 133 & 136 & 125 & $519(64.9)$ \\
\hline \multirow[t]{2}{*}{ Host } & Goat & 101 & 100 & 100 & 100 & $401(50.1)$ \\
\hline & Sheep & 99 & 100 & 100 & 100 & $399(49.9)$ \\
\hline \multirow[t]{2}{*}{ Ticks } & Present & 74 & 74 & 94 & 106 & $348(43.5)$ \\
\hline & Absent & 126 & 126 & 106 & 94 & $452(65.5)$ \\
\hline \multirow[t]{3}{*}{ Grazing } & Free & 85 & 87 & 37 & 91 & $300(37.5)$ \\
\hline & Semi & 48 & 48 & 52 & 48 & $196(24.5)$ \\
\hline & Zero & 67 & 65 & 111 & 61 & 304 (38.0) \\
\hline \multirow[t]{3}{*}{ Acaricides } & Regular & 68 & 70 & 57 & 70 & $265(33.1)$ \\
\hline & Irregular & 33 & 54 & 76 & 37 & $200(25.0)$ \\
\hline & No use & 99 & 76 & 67 & 93 & $335(41.9)$ \\
\hline
\end{tabular}

ribosomal RNA (SSU rRNA) of Anaplasma spp. (577 bp) [44] and 18S SSU rRNA gene of Theileria spp. (1098 bp) were used [4]. Species-specific primers were used to amplify an $870 \mathrm{bp}$ [45] fragment from the msp 4 gene of $A$. ovis and a 520 bp fragment from the small subunit ribosomal RNA gene of $T$. ovis [5]. PCR assays were performed with $250 \mathrm{ng}$ of genomic DNA using a MasterMix (including Taq polymerase, dNTP ( $0.4 \mathrm{mM}$ each) and buffer with $2 \mathrm{mM} \mathrm{MgCl} 2$; Thermo Fisher Scientific) and distilled water in a final volume of $25 \mu \mathrm{L}$. DNA samples positive for Theileria species, T. ovis, Anaplasma species and A. ovis were kindly provided by Prof. Dr. Munir Aktas at Firat University, Turkey and DNA of T. lestoquardi was provided by the Immunology and Cell Biology Research Center, Borstel, Germany and they were used as positive controls. Deionised water was used as negative control.

The thermoprofile that was used to detect Anaplasma spp., and Theileria spp. consisted of: denaturation for 7 min at $95^{\circ} \mathrm{C}$ followed by 30 cycles (denaturation at $95^{\circ} \mathrm{C}$ for $30 \mathrm{~s}$, annealing at $58.3{ }^{\circ} \mathrm{C}$ for $45 \mathrm{~s}$ and extension for $30 \mathrm{~s}$ at $72{ }^{\circ} \mathrm{C}$ ), followed by a final extension at $72{ }^{\circ} \mathrm{C}$ for $10 \mathrm{~min}$. Cycling conditions for the species-specific primers for the detection of T. ovis and A. ovis were the same as above, except that the number of cycles was increased to 35 and annealing temperature was $60^{\circ} \mathrm{C}$ for $1 \mathrm{~min}$. All PCR products were checked in $1.5 \%$ agarose gel stained with $0.5 \mu \mathrm{g} / \mathrm{mL}$ ethidium bromide and visualised under UV light (Bio-Rad laboratories, CA, USA).

\section{DNA sequencing and phylogenetic analyses}

PCR products were chosen, cleaned and sent to a commercial company (Macrogen, Inc., Seoul, South Korea) for Sanger sequencing in both directions $\left(3^{\prime}-5^{\prime}\right.$ and $\left.5^{\prime}-3^{\prime}\right)$ of the SSU rRNA gene of Theileria ovis and Theileria spp., and the msp4 gene of Anaplasma ovis.

Newly obtained sequences were compared with the NCBI database using the Basic Local Alignment Search Tool (BLAST) (https://blast.ncbi.nlm.nih.gov/Blast.cgi) and related sequences were obtained from the GenBank database (https:// www.ncbi.nlm.nih.gov/), with multiple alignments among sequences carried out with MUSCLE [14]. Phylogenetic analyses were conducted in MEGA, version X [33] using the top-ranking substitution models according to lowest BIC scores (Bayesian Information Criterion) and a bootstrap analysis with 1000 replicates. Phylogenetic trees were visualised and edited with iTOL (https://itol.embl.de/). Phylogenetic analyses of the msp4 gene from A. ovis and the SSU rRNA gene from T. ovis, were performed using the UPGMA method [55] with the Kimura 2-parameter model [29] and the Jukes-Cantor model [25], respectively. For SSU rRNA from $T$. annulata and T. lestoquardi, evolutionary histories were inferred using the Maximum Likelihood method with Kimura 2-parameter model [29]. Datasets of sequences obtained from GenBank and those generated in this study were trimmed to 854, 1056 and 482 bp for $m s p 4$ from $A$. ovis, SSU rRNA from $T$. annulata and T. lestoquardi, and SSU rRNA from $T$. ovis, respectively. Identity scores between the sequences were based on multiple alignments and calculated with the SIAS tool (http://imed. med.ucm.es/Tools/sias.html).

\section{Statistical analyses}

All statistical analyses for the present study were carried out using R software, version 3.5.1 (R Development Core Team, 2018). A chi-square test was used to compare prevalence between districts. For the determination of statistical significance and association between the pathogen prevalence and other independent variables, univariable and multivariable regression models (Generalized linear model univariable/Mix multivariable model) were used.

\section{Results \\ PCR-based prevalence of Theileria spp. and Anaplasma spp.}

Overall, 361/800 (45.1\%) animals were found to be infected by one of the haemopathogens, among them, 187/800 (23.37\%) were infected by Theileria spp., while 174/800 (21.75\%) were infected with Anaplasma spp. Prevalence of Theileria spp. and Anaplasma spp. coinfection was higher in sheep (14.5 and $13.87 \%$, respectively) than in goats $(8.87$ and $7.87 \%$, respectively) $(p<0.001)$ (Tables 2 and 3$)$. 
Table 2. Prevalence rates of Theileria spp., Theileria ovis, and Anaplasma ovis in small ruminants from four districts in Khyber Pakhtunkhwa. The $p$-value represents the results of Chi-square tests conducted to report the prevalence of a parasite among the sampling districts.

\begin{tabular}{|c|c|c|c|c|c|c|c|c|c|c|}
\hline \multirow[t]{2}{*}{ Districts } & \multicolumn{6}{|c|}{ Theileria spp. } & \multicolumn{3}{|c|}{ Anaplasma spp. } & \multirow[t]{2}{*}{ Total\% (CI) } \\
\hline & $\begin{array}{l}\text { T. ovis } \\
n(\%)\end{array}$ & CI 95\% & $p$-value & $\begin{array}{l}\text { Theileria } \\
\text { spp. n (\%) }\end{array}$ & CI 95\% & $p$-value & $\begin{array}{l}\text { A. ovis } \\
\text { n (\%) }\end{array}$ & CI $95 \%$ & $p$-value & \\
\hline Shangla & $21 / 200(10.5)$ & $6.62-15.60$ & 0.2 & $14 / 200(7)$ & $3.88-11.47$ & 0.7 & $32 / 200(16.0)$ & $11.21-21.83$ & 0.08 & $33.5(27.00-40.50)$ \\
\hline Swat & $32 / 200(16.0)$ & $11.21-21.83$ & & $19 / 200(9.5)$ & $5.82-11.44$ & & $48 / 200(24.0)$ & $18.26-30.53$ & & $49.5(42.37-56.64)$ \\
\hline Malakand & $35 / 200(17.5)$ & $12.50-23.49$ & & $21 / 200(10.5)$ & $6.62-15.60$ & & $52 / 200(26.0)$ & $20.07-32.66$ & & $54.0(46.83-61.05)$ \\
\hline Bajaur & $27 / 200(13.5)$ & $9.09-19.03$ & & $18 / 200(9)$ & $5.42-13.85$ & & $42 / 200(21.0)$ & $15.57-27.31$ & & $43.5(36.52-50.67)$ \\
\hline Total & $115 / 800(14.3)$ & & & $72 / 800(9)$ & & & $174 / 800(21.7)$ & & & $361 / 800(45.1)$ \\
\hline
\end{tabular}

$\mathrm{CI}=$ Confidence interval.

The highest overall prevalence of Theileria spp. and Anaplasma spp. was observed in the Malakand district 108/200 (54\%) followed by Swat 99/200 (49.5\%), Bajaur $87 / 200(43.5 \%)$ and Shangla 67/200 (33.5\%). Prevalence of pathogens varied non significantly $(p>0.05)$ when compared between the sampling districts (Table 2). All 187 samples positive for Theileria spp. (23.3\%) were analysed for Theileria ovis, and it was found that $14.3 \%$ (115/187) of animals (goats and sheep) were infected with $T$. ovis while $9 \%(72 / 187)$ were infected with other Theileria spp. (Table 2). Four Theileria spp.-positive samples were analysed by sequencing their 18 SSU rRNA gene and it was observed that at least two more Theileria species (T. lestoquardi and T. annulata) besides T. ovis, were present in these districts (Table 2, Fig. 4). All 174 blood samples that were found to be positive for Anaplasma spp. were also positive for A. ovis (21.7\%) (Table 2).

Of the 200 animals analysed from each district, prevalence of T. ovis and Theileria spp. were highest in Malakand district (17.5\% and 10.5\%), followed by Swat district (16\% and 9.5\%), Bajaur district (13.5\% and 9\%) and Shangla district (10.5\% and $7 \%)$. On the other hand, the highest prevalence of $A$. ovis was observed in Malakand district (26.0\%), while lowest prevalence was recorded in Shangla district (16.0\%) (Table 2).

\section{Anaplasma spp. risk factor analysis}

When risk factor data were analysed, it was observed that prevalence of Anaplasma spp. infection was higher in young animals $<6$ months than in older animals $\geq 6$ months of age: $100 / 800(12.5 \%)$ vs. $74 / 800(9.2 \%)$, respectively $(p<0.001)$. Based on different types of hosts, Anaplasma spp. was more common in sheep $(111 / 800 ; 13.8 \%)$ than in goats $(63 / 800$; $7.8 \%)(p<0.001)$. Anaplasma spp. $126 / 800 \quad(15.7 \%)$ prevalence was higher in animals that were not treated with acaricides, followed by irregularly treated animals (36/800; $4.5 \%)$, while the lowest prevalence $(12 / 800 ; 1.5 \%)$ was found in animals that were regularly treated with acaricides $(p<0.001)$. Grazing practices showed that the prevalence of Anaplasma spp. was higher $(116 / 800 ; 14.5 \%)$ in full-grazing practices, than irregular practices $(41 / 800 ; 5.1 \%)$, while the zero-grazing animals were least infected with Anaplasma spp. $(17 / 800 ; 2.1 \%)(p<0.001)$. Anaplasma spp. was highly prevalent in tick-infested goats and sheep, with a prevalence rate of 162/800 (20.2\%). However, goats and sheep not infested with ticks $(12 / 800 ; 1.5 \%)$ were found to be infected with Anaplasma spp.

\section{Univariable and multivariable risk factors regression analyses of Anaplasma spp. infection}

The potential risk factors associated with Anaplasma spp. infection based on univariable regression analyses were host $(\mathrm{OR}=0.48, \mathrm{CI}=0.34-0.68, p=0.001)$, age $(\mathrm{OR}=3.24$, $\mathrm{CI}=2.30-4.60, p=0.001)$, tick infestation $(\mathrm{OR}=3.46$, $\mathrm{CI}=0.31-11.11, p=0.001)$, grazing system $(\mathrm{OR}=0.34$, $\mathrm{CI}=0.26-0.42, p=0.001)$ and acaricide use $(\mathrm{OR}=3.30$, $\mathrm{CI}=2.57-4.33, p=0.001$ ). Similarly, the same factors (host, age, tick infestation, grazing system and acaricide use) were also statistically significant in the multivariable regression analyses with ORs of $0.83,1.33,3.04,0.56$. and 0.22 , along with $p=0.001$. Sex was only an associated potential risk factor on the multivariable regression analyses $(\mathrm{OR}=0.61 \mathrm{CI}=0.23$ 2.63, $p=0.001)$; Anaplasma spp. was more prevalent in females $(118 / 800 ; 14.7 \%)$ than males $(56 / 800 ; 7.0 \%)$.

\section{Theileria spp. risk factor analysis}

The risk factor analysis for Theileria spp. showed that young animals ( $<6$ months) had a higher infection rate $(114 / 800 ; 14.2 \%)$ than adult animals $\geq 6$ months of age $(73 / 800 ; 9.1 \%)(p<0.001)$, and the rate was higher in sheep $(116 / 800 ; 14.5 \%)$ than in goats $(71 / 800 ; 8.8 \%)(p<0.001)$ (Table 3). Theileria prevalence $(134 / 800 ; 16.7 \%)$ was higher in animals with no acaricide treatment, followed by irregularly treated animals (40/800; 5\%), and animals that were regularly treated with acaricides presented the lowest prevalence $(13 / 800 ; 1.6 \%)(p<0.001)$. Animals in full-grazing systems had higher prevalence $(125 / 800 ; 15.6 \%)$ than those in a semi-grazing $(40 / 800 ; 5 \%)$ or zero-grazing systems (13/800; $2.6 \%)(p<0.001)$. Animals infested with ticks had higher prevalence $(174 / 800 ; 21.7 \%)$ compared with animals with no ticks $(14 / 800 ; 1.7 \%)(p<0.001)$.

\section{Univariable and multivariable regression analyses for Theileria spp. infection risk factors}

The potential risk factors associated with Theileria spp. infection based on univariable regression analyses were host 
Table 3. Results of univariable and multivariable logistic regression analysis of Theileria spp. and Anaplasma ovis PCR-positive infections in sheep and goats in Northern Pakistan.

\begin{tabular}{|c|c|c|c|c|c|c|c|c|c|c|c|c|}
\hline \multirow[t]{3}{*}{ Risk factors } & \multicolumn{6}{|c|}{ Theileria spp. } & \multicolumn{6}{|c|}{ Anaplasma ovis } \\
\hline & \multicolumn{2}{|c|}{ Prevalence } & \multicolumn{2}{|l|}{ Univariable } & \multicolumn{2}{|c|}{ Multivariable } & \multicolumn{2}{|c|}{ Prevalence } & \multicolumn{2}{|l|}{ Univariable } & \multicolumn{2}{|c|}{ Multivariable } \\
\hline & Positive (\%) & CI 95\% & OR $(95 \% \mathrm{CI})$ & $p$-value & OR $(95 \% \mathrm{CI})$ & $p$-value & Positive (\%) & CI 95\% & OR $(95 \% \mathrm{CI})$ & $p$-value & OR $(95 \% \mathrm{CI})$ & $p$-value \\
\hline \multicolumn{13}{|l|}{ Host } \\
\hline Goat & $71(8.8)$ & $6.83-10.76$ & $0.52(0.37-0.73)$ & 0.001 & $0.42(0.27-0.65)$ & 0.001 & $63(7.8)$ & $6.01-9.74$ & $0.48(0.34-0.68)$ & 0.001 & $0.83(0.22-3.86)$ & 0.001 \\
\hline Sheep & $116(14.5)$ & $12.06-16.33$ & & & & & $111(13.8)$ & & & & & \\
\hline \multicolumn{13}{|l|}{ Sex } \\
\hline Male & $59(7.3)$ & $5.55-9.18$ & $0.8(0.56-1.14)$ & 0.242 & $0.54(0.33-0.85)$ & 0.001 & $56(7.0)$ & $5.23-8.76$ & $0.84(0.58-1.20)$ & 0.35 & $0.61(0.23-2.63)$ & 0.001 \\
\hline Female & $128(16.0)$ & $13.45-18.54$ & & & & & $118(14.7)$ & $12.29-17.20$ & & & & \\
\hline \multicolumn{13}{|l|}{ Age } \\
\hline$<6$ months & $114(14.2)$ & $11.71-16.53$ & $3.7(2.71-5.36)$ & 0.001 & $3.77(2.44-5.87)$ & 0.001 & $100(12.5)$ & $10.20-14.79$ & $3.24(2.30-4.60)$ & 0.001 & $1.33(0.22-5.88)$ & 0.001 \\
\hline$\geq 6$ months & $73(9.1)$ & $7.24-11.25$ & & & & & $74(9.2)$ & $7.24-11.25$ & & & & \\
\hline \multicolumn{13}{|c|}{ Tick infestation } \\
\hline Present & $174(21.7)$ & $18.89-24.61$ & $3.43(0.29-11.75)$ & 0.001 & $20.9(11.01-42.63)$ & 0.001 & $162(20.2)$ & $17.46-23.03$ & $3.46(0.31-11.11)$ & 0.001 & $3.04(0.34-8.85)$ & 0.001 \\
\hline Absent & $14(1.7)$ & $0.84-2.65$ & & & & & $12(1.5)$ & $0.65-2.35$ & & & & \\
\hline Full grazing & $125(15.6)$ & $13.11-18.14$ & & & & & $116(14.5)$ & $12.06-16.93$ & & & & \\
\hline Semi & $40(5)$ & $3.48-6.51$ & $0.32(0.26-0.41)$ & 0.001 & $0.57(0.42-0.75)$ & 0.001 & $41(5.1)$ & $3.57-6.62$ & $0.34(0.26-0.42)$ & 0.001 & $0.56(0.14-3.88)$ & 0.001 \\
\hline Zero & $21(2.6)$ & $1.51-3.73$ & & & & & $17(2.1)$ & $1.10-3.09$ & & & & \\
\hline \multicolumn{13}{|l|}{ Acaricides } \\
\hline Regular & $13(1.6)$ & $0.73-2.46$ & & & & & $12(1.5)$ & $1.41-3.58$ & & & & \\
\hline Irregular & $40(5.0)$ & $3.48-6.51$ & $3.30(2.58-4.29)$ & 0.001 & $1.25(0.88-1.01)$ & 0.18 & $36(4.5)$ & $3.06-5.93$ & $3.30(2.57-4.33)$ & 0.001 & $0.22(0.17-1.29)$ & 0.19 \\
\hline No use & $134(16.7)$ & $14.16-19.33$ & & & & & $126(15.7)$ & $13.17-18.22$ & & & & \\
\hline
\end{tabular}

$\mathrm{OR}=$ Odds ratio, $\mathrm{CI}=$ Confidence interval. Semi $=$ semi-grazing system.

$(\mathrm{OR}=0.52, \mathrm{CI}=0.37-0.73, p=0.001)$, age $(\mathrm{OR}=3.7$, $\mathrm{CI}=2.71-5.36, p=0.001)$, grazing system $(\mathrm{OR}=0.32$, $\mathrm{CI}=0.26-0.41, p=0.001)$, tick infestation $(\mathrm{OR}=3.43$, $\mathrm{CI}=0.29-11.75, p=0.001)$ and acaricide use $(\mathrm{OR}=3.30$, $\mathrm{CI}=2.58-4.29, p=0.001)$. The same risk factors such as host $(\mathrm{OR}=0.42, \mathrm{CI}=0.27-0.65, p=0.001$, age $(\mathrm{OR}=3.77$, $\mathrm{CI}=2.44-5.87, p=0.001)$, tick infestation $(\mathrm{OR}=20.9$, $\mathrm{CI}=11.01-42.63, p=0.001)$, and grazing system $(\mathrm{OR}=0.32, \mathrm{CI}=0.26-0.41, p=0.001)$ were associated with Theileria spp. infection in the multivariable regression analyses. Sex was associated as a potential risk factor only on the multivariable regression analyses $(\mathrm{OR}=0.54, \mathrm{CI}=0.33-0.85$, $p=0.001)$, with a prevalence of $128 / 800(16 \%)$ in females and $59 / 800(7.3 \%)$ in males.

\section{Phylogenetic analysis}

The msp4 gene sequences from $A$. ovis were deposited in GenBank under accession numbers MT311200, MT311201, MT311202, and MT311203. The small subunit ribosomal RNA gene sequences from $T$. ovis were deposited under accession numbers MT318208, MT318209, and MT318210, from T. lestoquardi under accession number MT318171, and from T. annulata under accessions numbers MT318158, MT318159 and MT318158. Anaplasma ovis from Pakistan clearly clustered with other A. ovis sequences (Fig. 2); however, based on a multiple alignment (data not shown), the sequences present nucleotide differences between them, suggesting that different strains are circulating in the analysed areas.

Concerning the $T$. ovis PCR samples that were amplified with the specific primers for $T$. ovis, three sequences were phylogenetically analysed to reveal the relationship between SSU rRNA gene sequences and other Theileria species sequences obtained from GenBank. According to the tree, sequences MT318208, MT318209 and MT318210 distinctly clustered with T. ovis sequences (Fig. 3) and they shared $100 \%$ identity between them and with another $T$. ovis strain isolated from Pakistan (accession numbers: MN922940 and MN922939), Egypt (accession numbers: MN625903 and MN625887), Iraq (accession number: MN560042), Turkey (accession number: MN493111), India (accession numbers: MH819509 and MH819510), Tanzania (accession number: MG725961), and South Korea (accession number: FJ668373) based on a multiple alignment (data not shown).

Four DNA sequences that were found to be positive following their amplification with generic primers for Theileria spp. were phylogenetically analysed to reveal the relationship between their SSU rRNA gene sequences and other Theileria species sequences obtained from GenBank. One of these sequences (MT318171) clustered with T. lestoquardi sequences. The other three sequences (MT318158, MT318159 and MT318160) clustered with T. annulata sequences (Fig. 4).

\section{Discussion}

To our knowledge, this is the first study conducted for molecular detection and phylogenetic analysis of tick-borne pathogens in small ruminants from four different districts of Khyber Pakhtunkhwa, northern Pakistan (Malakand, Swat, Bajaur and Shangla). This study determined the status of tickborne diseases in small ruminants from these areas that were 


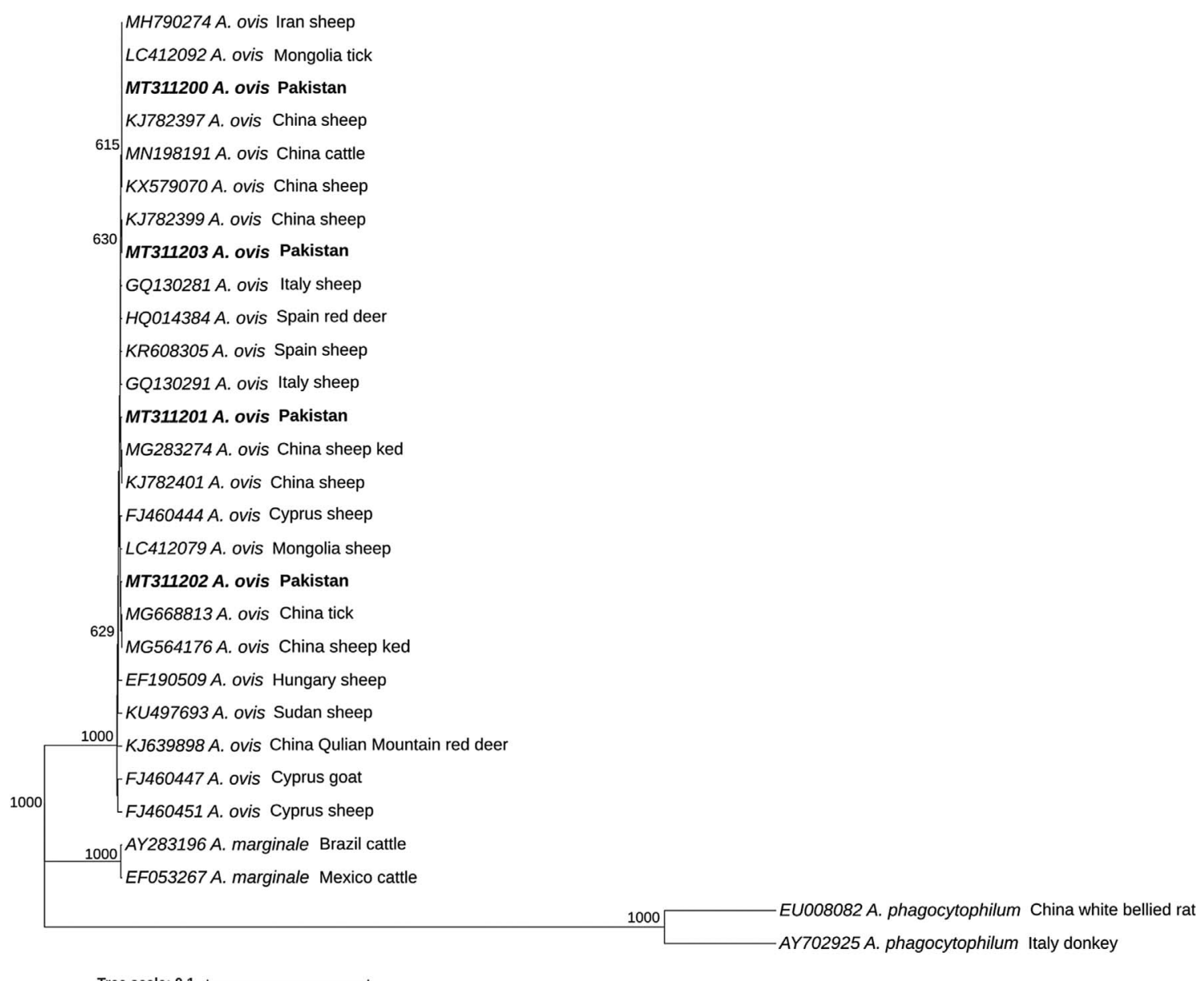

Figure 2. Phylogenetic analysis of Anaplasma ovis based on the msp4 gene. The sequences determined in this study are shown in bold font. Numbers at the nodes represent the number of occurrences of clades in 1000 bootstrap replications of the taxa. Phylogenetic analysis was performed using MEGA 10 software. The msp4 gene sequences of Anaplasma marginale (EF052297 and AY283196) and Anaplasma phagocytophilum (EU008082 and AY702925) were used as the outgroup.

affected by war for more than 20 years. The geographical location of the study area is very important as its boundaries are aligned with two neighbouring countries, Iran and China.

Three Theileria spp. ( $T$. ovis, T. lestoquardi and T. annulata) and one Anaplasma sp. (A. ovis) of haemopathogens were identified both in goats and sheep.

\section{Prevalence}

The study revealed an overall prevalence of $45.1 \%$ for both pathogens in small ruminants from northern Pakistan of which 23.3\% were Theileria, which is consistent with previous studies (17.4-31.2\%) from Bannu, Tank and Dera Ismail Khan districts in southern Khyber Pakhtunkhwa [58] and elsewhere (17.8 and $17.2 \%$ in Turkey and China, respectively) [8, 64]. However, some studies reported contrasting prevalences; for example, a study by Nasreen et al. [38] in the Dir District, Lower KP showed a prevalence of $53.5 \%$, while Durrani et al. [13] reported a prevalence of 35\% from Lahore with PCR. Similarly, a lower prevalence (3.47\%) was reported in Southern Punjab [17], 6\% in Kohat and Peshawar [13], while Saeed et al. [49] reported a prevalence of 3\% in Kohat and Peshawar. These differences may be due to different geographical areas and climatic conditions [13]. In our study, T. ovis was more prevalent than Theileria spp. which is consistent with previously studies from different parts of the country $[38,47]$ and elsewhere [60]. Theileria annulata is mainly a pathogen of cattle; experimental infections of sheep and goats with $T$. annulata cause mild symptoms with no development of piroplasm [35]. It is necessary to study the clinical signs of $T$. annulata on naturally infected animals to determine the clinic importance of this infections and the role of animals as a reservoir for the pathogen in the area.

Similarly, our results showed an overall prevalence of 21.7\% for Anaplasma spp. which is similar to previous findings in Mardan District, Khyber Pakhtunkhwa, Pakistan with 23.33\% using cELISA [37]. However, the prevalence we estimated was slightly lower than those previously reported by Hussein et al [22] (56.25\%) and by Shah et al. [54] (40.7\%) in Karak and Peshawar, respectively.

The overall prevalence of $A$. ovis was estimated to be $21.7 \%$, which falls within the range of $11.5-87.4 \%$ previously reported across the world by other authors $[10,12,24,36$, $46,54]$. 


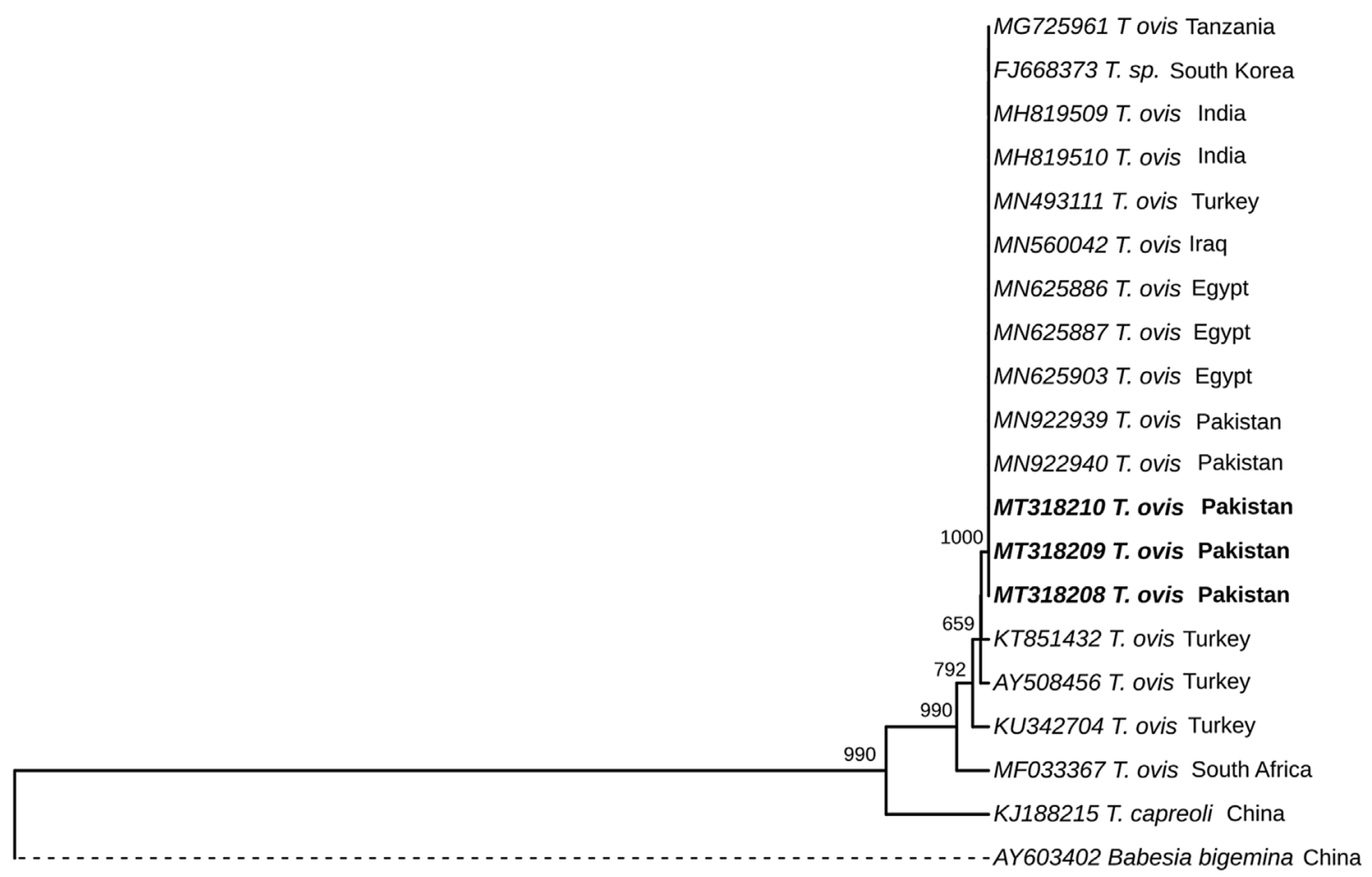

Tree scale: 0.01

Figure 3. Phylogenetic analysis of Theileria ovis based on the SSU rRNA gene. The sequences generated in this study are shown in bold font. Numbers at the nodes represent the number of occurrences of clades in 1000 bootstrap replications of the taxa. Phylogenetic analysis was performed using MEGA 10 software. The SSU rRNA gene sequences of Babesia bigemina (AY603402) and Theileria capreoli (KJ188215) were used as the outgroup.

The PCR results revealed in sheep and goats an Anaplasma ovis prevalence rate of 16.0, 21.0, 24.0 and 26.0\% for Shangla, Bajuar, Swat and Malakand districts, respectively. These prevalences are lower than those reported from Turkey $(71.32 \%)$ and Pakistan $(56.25 \%)[6,22]$, but higher than those reported in China (11.7\%) and the United States (14\%) in sheep $[18,42]$. Similarly, higher molecular Anaplasma prevalences have been reported in Iranian goats (38.9\%) [44] and (63.7\%) [2]. These variations may be due to geographical location, different diagnostic methods used, animal breeds with different susceptibility to pathogens, proximity of veterinarian services, seasons of sample collection, climatic differences, tick infestation intensity, and absence of mechanical and biological vectors in that particular area.

In the current study, the prevalence of Theileria and Anaplasma was significantly higher in sheep (14.5 and $13.9 \%$ ) than in goats ( 8.8 and $7.9 \%$ ), which may be due to the nature of their skin. The skin of goats is thinner and shows resistance to the attachment of ticks due to its smooth aspect, while sheep have wool and ticks become easily entangled making sheep more susceptible to infection $[21,47,54]$. The lower prevalence in goats than in sheep could also be due to the ability of goats to pasture in areas that are steep and poorly accessible, where there are far fewer opportunities for contact with ticks that feed on other animals during their life cycle [3].

Our results showed that animals younger than 6 months of age had higher levels of theileriosis infection. This may be due to an underdeveloped immune system, deficient grooming, or another risk factor that was not analysed in this study, such as vaccination or weaning. It has previously been reported that goats 3-6 months of age had a higher risk of infection than other age groups, with a reported infection rate of $19.2 \%$ (95\% CI: $16.4-22.0 \%$ ) [60].

In the present study, a higher prevalence of Anaplasma sp. was observed in young animals compared to adults; similar findings were also reported previously [20, 54]. Similarly, sex-specific data revealed that females showed a higher prevalence compared to males, findings consistent with those previously reported from different regions of Pakistan $[7,13,65]$ and Nigeria [26]. Differences may be due to several factors, including the fact that females are frequently under stress: pregnancy and lactation [7].

\section{Risk factors}

The risk factors of host, age and grazing system were statistically significant for Theileria infection by both univariate and multivariate analysis. Grazing animals are at a higher risk than non-grazing animals as a result of greater exposure to tick infestation since they are exophilic [15]. Interestingly, acaricide application was statistically significant in the case of univariable analysis; however, its effect was non-significant in the multivariable analysis. Similar results were reported by Nasreen et al [38]. Risk factors such as host, age and grazing system were statistically significant for Anaplasma in both multivariable and univariable analysis. Obviously, animals infested with 


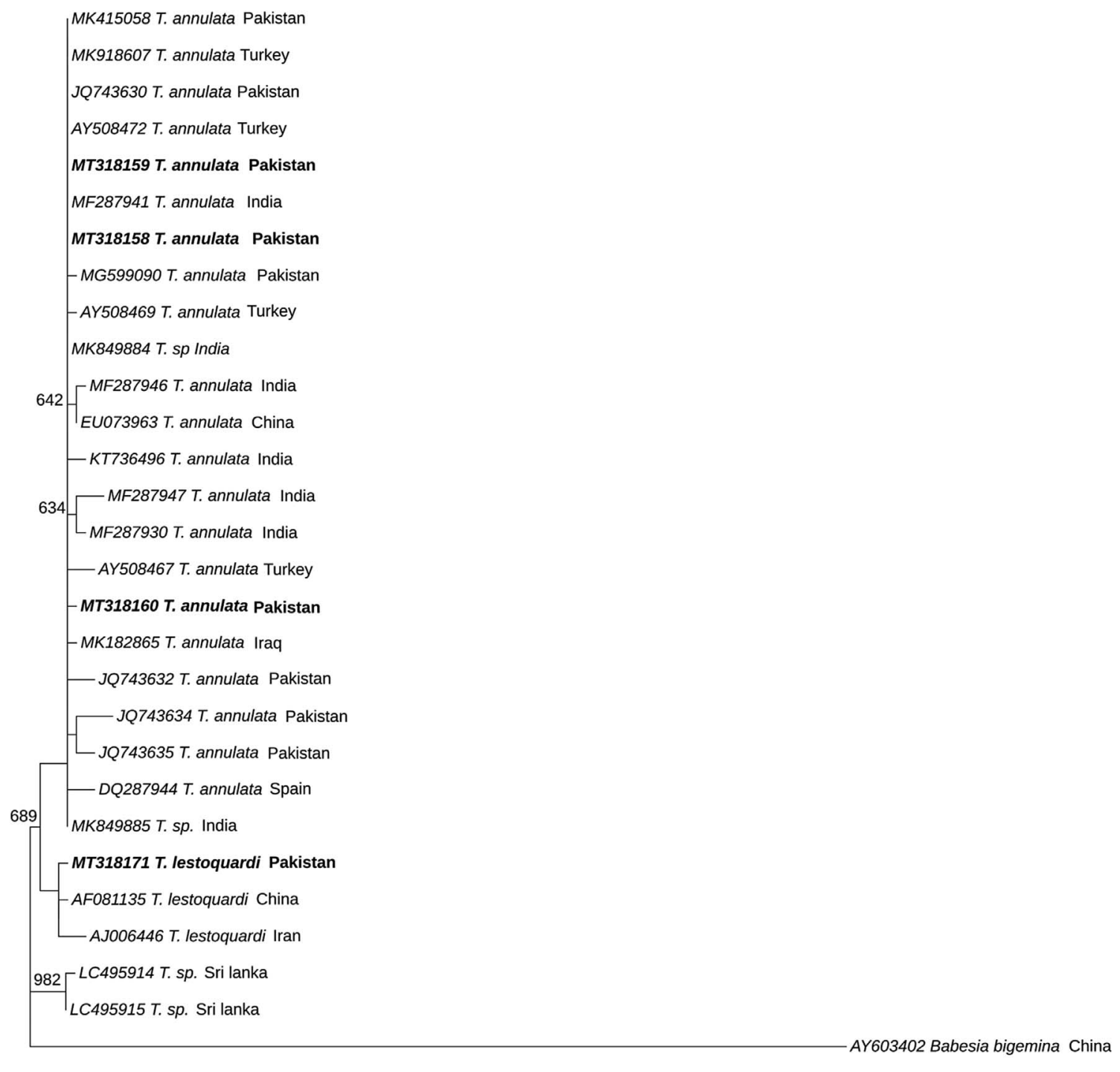

Tree scale: 0.01

Figure 4. Phylogenetic analysis of Theileria annulata and Theileria lestoquardi based on the SSU rRNA gene. The sequences in bold font are from this study. The numbers at the nodes represent the number of occurrences of the clades in 1000 bootstrap replications of the taxa. Phylogenetic analysis was performed using MEGA 10 software. The SSU rRNA gene sequence of Babesia bigemina (AY603402) was used as the outgroup.

larger numbers of ticks are at greater risk of both Anaplasma and Theileria infections [17, 59].

\section{Phylogenetic analyses}

Phylogenetic analyses revealed that Anaplasma ovis detected in the four different districts are genetically different, indicating that different genotypes are circulating in these regions. The sequences shared identity scores of $99.6 \%-99.9 \%$ between themselves and $99 \%-100 \%$ with already reported $m s p 4$ sequences of A. ovis identified in several host species from nine countries and that were used in this study. Genetic variability has also been reported for A. ovis using this marker [19, 44]. Isolate MT311200 from Pakistan was identical to sequences from China (accession numbers: K579070, MN198191 and KJ782397), Iran (accession number: MH790274) and Mongolia (accession number: LC412092). Sequence MT311203 was identical to an isolate from China (accession number: KJ782399), while MT311201 and MT311202 were distinct variants. The results of this study highlight the need for detailed investigations to characterise the regional genetic diversity of A. ovis in Pakistan and correlate it to virulence.

Three Theileria species were identified in the isolates analysed, namely, T. ovis, T. annulata and T. lestoquardi. No variation was found between the SSU rRNA gene sequences of $T$. ovis from this study and the T. ovis obtain from Africa (accession number: MG725961) and Asia (accession numbers: MH819510, MH819509, MN560042, MN493111, MN625903, MN625887 and MN625887). Furthermore, as shown in the phylogenetic tree, MT318208, MT318209 and MT318210 T. ovis SSU rRNA sequences also clustered with T. ovis sequences from Pakistan (accession numbers: MN922939 and MN922940) deposited in GenBank. Similar results have shown few or no variations between $T$. ovis isolates 
obtained from different countries, such as Kenya, Sudan and Tanzania, [36] suggesting that similar genotypes are circulating in several regions of the world.

In order to confirm and explore the sequence diversity of the $T$. annulata and T. lestoquardi sequences obtained in this study, a phylogenetic tree was constructed using the SSU rRNA gene. The $T$. lestoquardi sequence (accession number: MT318171) from this study clustered with $T$. lestoquardi sequences from China (accession number: AF081135) and Iran (accession number: AJ006446), in a separate clade. On the other hand, T. annulata showed sequence variants between the samples analysed in the present study. With respect to the T. annulata isolates from Pakistan and sequences reported herein, it was observed that the sequence with accession number MT318158 was identical to MT318159, while MT318160 shared an identity score of $99.90 \%$ with the other two sequences. Theileria annulata amplicons in the present study clustered with isolates of $T$. annulata from Pakistan, India, Spain, Turkey, Iraq and China, and they were clearly separated from the T. lestoquardi clade. Theileria annulata variants were previously reported from isolates from Pakistan districts along the India-Pakistan border [28]. These isolates also displayed variation from the isolates reported herein, highlighting the importance of addressing these variants in Pakistan in future studies. To evaluate the influence of legal and illegal animal trade and mobility, additional studies with larger sample sizes are required.

\section{Conclusion}

This study demonstrated that A. ovis, T. ovis, T. annulata and T. lestoquardi are present in Northern Pakistan. Theileria spp. and T. ovis are more prevalent than Anaplasma spp. and these infections are more prevalent in sheep than in goats. Univariable analysis of risk factors showed that host, age, grazing system and acaricide treatment were significant risk factors $(p<0.05)$. Multivariable analysis of risk factors revealed that host, sex, age, tick infestation and grazing system were significant risk factors $(p<0.05)$ for both pathogens. Additionally, it was found that different genotypes of $A$. ovis and $T$. annulata are circulating in the field.

\section{Recommendation for future research}

This study emphasises the need to conduct future research assessing the epidemiology of tick and tick-borne diseases across different agro-ecological zones, in different seasons and under various production systems to have a clear picture of the diseases and risk factors, which is very important to control these diseases in Pakistan.

\section{Competing financial interests}

The authors declare that they have no competing financial interests.

Acknowledgements. We acknowledge the support of Dr. Stephen Wilken for English proofreading an early version of this paper, as well as the farmers and veterinarians who helped us by providing blood samples. This research did not receive any specific grant from funding agencies in the public, commercial, or not-for-profit sectors.

\section{References}

1. Abdullah DA, Ali FF, Jasim AY, Ola-Fadunsin SD, Gimba FI, Ali MS. 2020. Clinical signs, prevalence, and hematobiochemical profiles associated with Anaplasma infections in sheep of North Iraq. Veterinary World, 13, 1524-1527.

2. Ahmadi-Hamedani M, Khaki Z, Rahbari S, Kazemi B, Bandehpour M. 2009. Molecular identification of anaplasmosis in goats using a new PCR-RFLP method. Iranian Journal of Veterinary Research, 10, 367-372.

3. Alessandra T, Santo C. 2012. Tick-borne diseases in sheep and goats: clinical and diagnostic aspects. Small Ruminant Research, 106, S6-S11.

4. Allsopp BA, Baylis HA, Allsopp MT, Cavalier-Smith T, Bishop RP, Carrington DM, Sohanpal B, Spooner P. 1993. Discrimination between six species of Theileria using oligonucleotide probes which detect small subunit ribosomal RNA sequences. Parasitology, 107(Pt 2), 157-165.

5. Altay K, Dumanli N, Holman PJ, Aktas M. 2005. Detection of Theileria ovis in naturally infected sheep by nested PCR. Veterinary Parasitology, 127, 99-104.

6. Altay K, Dumanlı N, Aktaş M, Özübek S. 2014. Survey of anaplasma infections in small ruminants from east part of Turkey. Kafkas Üniversitesi Veteriner Fakültesi Dergisi, 20, $1-4$.

7. Atif FA, Khan MS, Iqbal HJ, Arshad GM, Ashraf E, Ullah S. 2012. Prevalence of Anaplasma marginale, Babesia bigemina and Theileria annulata infections among cattle in Sargodha District, Pakistan. African Journal of Agricultural Research, 7, 3302-3307.

8. Aydin MF, Aktas M, Dumanli N. 2013. Molecular identification of Theileria and Babesia in sheep and goats in the Black Sea Region in Turkey. Parasitology Research, 112, 2817-2824.

9. Bishop R, Musoke A, Morzaria S, Gardner M, Nene V. 2004. Theileria: intracellular protozoan parasites of wild and domestic ruminants transmitted by ixodid ticks. Parasitology, 129(Suppl), S271-283.

10. Cabezas-Cruz A, Gallois M, Fontugne M, Allain E, Denoual M, Moutailler S, Devillers E, Zientara S, Memmi M, Chauvin A, Agoulon A, Vayssier-Taussat M, Chartier C. 2019. Epidemiology and genetic diversity of Anaplasma ovis in goats in Corsica, France. Parasites \& Vectors, 12, 3.

11. Demessie Y, Derso S. 2015. Tick borne hemoparasitic diseases of ruminants: a review. Advances in Biological Research, 9, 210-224.

12. Djiba ML, Mediannikov O, Mbengue M, Thiongane Y, Molez J-F, Seck MT, Fenollar F, Raoult D, Ndiaye M. 2013. Survey of Anaplasmataceae bacteria in sheep from Senegal. Tropical Animal Health and Production, 45, 1557-1561.

13. Durrani S, Khan Z, Khattak RM, Andleeb M, Ali M, Hameed H, Taqddas A, Faryal M, Kiran S, Anwar H, Riaz M, Sajid M, Sheikh RS, Ali M, Iqbal F. 2012. A comparison of the presence of Theileria ovis by PCR amplification of their SSU rRNA gene in small ruminants from two provinces of Pakistan. Asian Pacific Journal of Tropical Disease, 2, 43-47.

14. Edgar RC. 2004. MUSCLE: multiple sequence alignment with high accuracy and high throughput. Nucleic Acids Research, 32, 1792-1797.

15. Eliyas M, Mannan M, Hashem M, Debnath N, Miazi O, Mohiuddin C, Kashem M, Islam M, Elahi M. 2011. An 
epidemiological survey on investigation of tick infestation in cattle at Chittagong District, Bangladesh. African Journal of Microbiology Research, 5, 346-352.

16. Enkhtaivan B, Narantsatsral S, Davaasuren B, Otgonsuren D, Amgalanbaatar T, Uuganbayar E, Zoljargal M, Myagmarsuren P, Suganuma K, Molefe NI, Sivakumar T, Inoue N, Battur B, Battsetseg B, Yokoyama N. 2019. Molecular detection of Anaplasma ovis in small ruminants and ixodid ticks from Mongolia. Parasitology International, 69, 47-53.

17. Fatima M, Saeed S, Shaikh RS, Ali M, Iqbal F. 2015. A study on molecular detection of Theileria lestoquardi by PCR amplification in apparently healthy small ruminants from five districts of Southern Punjab. Pakistan Journal of Zoology, 47, 441-446.

18. de la Fuente J, Atkinson MW, Hogg JT, Miller DS, Naranjo V, Almazán C, Anderson N, Kocan KM. 2006. Genetic characterization of Anaplasma ovis strains from bighorn sheep in Montana. Journal of Wildlife Diseases, 42, 381-385.

19. Gubbels MJ, Hong Y, van der Weide M, Qi B, Nijman IJ, Guangyuan L, Jongejan F. 2000. Molecular characterization of the Theileria buffeli/orientalis group. International Journal for Parasitology, 30, 943-952.

20. Hakimi H, Sarani A, Takeda M, Kaneko O, Asada M. 2019. Epidemiology, risk factors, and co-infection of vector-borne pathogens in goats from Sistan and Baluchestan province, Iran. PLoS One, 14, e0218609.

21. Hosseini-Vasoukolaei N, Oshaghi MA, Shayan P, Vatandoost H, Babamahmoudi F, Yaghoobi-Ershadi MR, Telmadarraiy Z, Mohtarami F. 2014. Anaplasma infection in ticks, livestock and human in Ghaemshahr, Mazandaran Province, Iran. Journal of Arthropod-Borne Diseases, 8, 204-211.

22. Hussain M, Junaid A, Gul R, Jamal M, Ahmed I, Talpur M, Rahim R, Fatima M, Munir S. 2017. First detection on prevalence of Anaplasma marginale in sheep and goat in Karak District, Pakistan. Asian Pacific Journal of Tropical Disease, 79, 531-535.

23. Jabbar A, Abbas T, Sandhu Z-D, Saddiqi HA, Qamar MF, Gasser RB. 2015. Tick-borne diseases of bovines in Pakistan: major scope for future research and improved control. Parasites \& Vectors, 8, 283.

24. Jalali SM, Khaki Z, Kazemi B, Bandehpour M, Rahbari S, Jalali MR, Yasini SP. 2013. Molecular detection and identification of Anaplasma species in sheep from Ahvaz, Iran. Iranian Journal of Veterinary Research, 14, 50-56.

25. Jukes TH, Cantor CR. 1969. CHAPTER 24 - Evolution of protein molecules, in Mammalian protein metabolism, Munro HN, Editor. Academic Press: New York. p. 21-132.

26. Kamani J, Sannusi A, Egwu OK, Dogo GI, Tanko TJ, Kemza S, Tafarki AE, Gbise DS. 2010. Prevalence and significance of haemoparasitic infections of cattle in North-Central, Nigeria. Veterinary World, 3, 445-448.

27. Khan M, Khan M, Ahmad S, Mahmood S. 2007. Genetic resources and diversity in Pakistani sheep. International Journal of Agriculture and Biology, 9, 941-944.

28. Khan MK, He L, Hussain A, Azam S, Zhang W-J, Wang L-X, Zhang Q-L, Hu M, Zhou Y-Q, Zhao J. 2013. Molecular epidemiology of Theileria annulata and identification of $18 \mathrm{~S}$ rRNA gene and ITS regions sequences variants in apparently healthy buffaloes and cattle in Pakistan. Infection, Genetics and Evolution: Journal of Molecular Epidemiology and Evolutionary Genetics in Infectious Diseases, 13, 124-132.

29. Kimura M. 1980. A simple method for estimating evolutionary rates of base substitutions through comparative studies of nucleotide sequences. Journal of Molecular Evolution, 16, 111120.
30. Kocan KM, de la Fuente J, Guglielmone AA, Meléndez RD. 2003. Antigens and alternatives for control of Anaplasma marginale infection in cattle. Clinical Microbiology Reviews, 16, 698-712.

31. Kocan KM, de la Fuente J, Blouin EF, Coetzee JF, Ewing SA. 2010. The natural history of Anaplasma marginale. Veterinary Parasitology, 167, 95-107.

32. Kocan KM, Busby AT, Allison RW, Breshears MA, Coburn L, Galindo RC, Ayllón N, Blouin EF, de la Fuente J. 2012. Sheep experimentally infected with a human isolate of Anaplasma phagocytophilum serve as a host for infection of Ixodes scapularis ticks. Ticks and Tick-Borne Diseases, 3, 147-153.

33. Kumar S, Stecher G, Li M, Knyaz C, Tamura K. 2018. MEGA $\mathrm{X}$ : molecular evolutionary genetics analysis across computing platforms. Molecular Biology and Evolution, 35, 1547-1549.

34. Li Y, Luo J, Guan G, Ma M, Liu A, Liu J, Ren Q, Niu Q, Lu B, Gao J, Liu Z, Dang Z, Tian Z, Zhang B, He Z, Bai Q, Yin H. 2009. Experimental transmission of Theileria uilenbergi infective for small ruminants by Haemaphysalis longicornis and Haemaphysalis qinghaiensis. Parasitology Research, 104, 1227-1231.

35. Li Y, Liu Z, Yang J, Chen Z, Guan G, Niu Q, Zhang X, Luo J, Yin H. 2014. Infection of small ruminants and their red blood cells with Theileria annulata schizonts. Experimental Parasitology, 137, 21-24.

36. Liu Z, Ma M, Wang Z, Wang J, Peng Y, Li Y, Guan G, Luo J, Yin H. 2012. Molecular survey and genetic identification of Anaplasma species in goats from central and southern China. Applied and Environmental Microbiology, 78, 464-470.

37. Nasreen KS, Khan A, Niaz S, Akhtar N. 2016. Serodiagnosis and haematological effect of Anaplasmosis in goats and sheep of District Mardan, Khyber Pakhtunkhwa, Pakistan. World Journal of Zoology, 11, 67-80.

38. NasreenKhan A, Niaz S, Hassan Shah M, Khan A, Ahmed H, Khattak I, Zeb J, Naeem H, Hassan MA, Ulucesme MC, Ozubek S, Aktas M. 2020. Molecular detection of small ruminant piroplasmosis and first report of Theileria luwenshuni (Apicomplexa: Theileridae) in small ruminants of Pakistan. Experimental Parasitology, 212, 107872.

39. Naz S, Maqbool A, Ahmed S, Ashraf K, Ahmed N, Saeed K, Latif M, Iqbal J, Ali Z, Shafi K, Nagra IA. 2012. Prevalence of theileriosis in small ruminants in Lahore-Pakistan. Journal of Veterinary and Animal Sciences (Lahore), 2, 16-20.

40. Peng Y, Wang K, Zhao S, Yan Y, Wang H, Jing J, Jian F, Wang R, Zhang L, Ning C. 2018. Detection and phylogenetic characterization of Anaplasma capra: an emerging pathogen in sheep and goats in China. Frontiers in Cellular and Infection Microbiology, 8, 283.

41. PES. 2018. Pakistan economic survey (2018-19), Chapter 2. Pakistan: Agriculture. p. 11-33.

42. Qiu H, Kelly PJ, Zhang J, Luo Q, Yang Y, Mao Y, Yang Z, Li J, Wu H, Wang C. 2016. Molecular detection of Anaplasma spp. and Ehrlichia spp. in ruminants from twelve provinces of China. Canadian Journal of Infectious Diseases and Medical Microbiology, 2016, 9183861.

43. R Core Team. 2013. R: A language and environment for statistical computing. Vienna, Austria: R Foundation for Statistical Computing. URL http://www.R-project.org/.

44. Razmi GR, Dastjerdi K, Hossieni H, Naghibi A, Barati F, Aslani MR. 2006. An epidemiological study on Anaplasma infection in cattle, sheep, and goats in Mashhad Suburb, Khorasan Province, Iran. Annals of the New York Academy of Sciences, 1078, 479-481.

45. Razzaq F, Khosa T, Ahmad S, Hussain M, Saeed Z, Khan MA, Shaikh RS, Ali M, Iqbal F. 2015. Prevalence of Anaplasma 
phagocytophilum in horses from Southern Punjab (Pakistan). Tropical Biomedicine, 32, 233-239.

46. Renneker S, Abdo J, Salih DEA, Karagenç T, Bilgiç H, Torina A, Oliva AG, Campos J, Kullmann B, Áhmed J, Seitzer U. 2013. Can Anaplasma ovis in small ruminants be neglected any longer? Transboundary and Emerging Diseases, 60(Suppl 2), 105-112.

47. Riaz M, Tasawar Z. 2017. Identification of Theileria species (Theileria ovis and Theileria lestoquardi) BY. Journal of Animal and Plant Sciences, 27, 809-818.

48. Ristic M. 1968. Anaplasmosis, in Diseases of Man and Animals. Academic Press Inc.: New York, NY. p. 474-537.

49. Roberts TJ. 1997. The mammals of Pakistan. Karachi, Pakistan: Oxford University Press.

50. Saeed S, Jahangir M, Fatima M, Shaikh RS, Khattak RM, Ali M, Iqbal F. 2015. PCR based detection of Theileria lestoquardi in apparently healthy sheep and goats from two districts in Khyber Pukhtoon Khwa (Pakistan). Tropical Biomedicine, 32, 225-232.

51. Schnittger L, Yin H, Jianxun L, Ludwig W, Shayan P, Rahbari S, Voss-Holtmann A, Ahmed JS. 2000. Ribosomal smallsubunit RNA gene-sequence analysis of Theileria lestoquardi and a Theileria species highly pathogenic for small ruminants in China. Parasitology Research, 86, 352-358.

52. Schnittger L, Yin H, Gubbels MJ, Beyer D, Niemann S, Jongejan F, Ahmed JS. 2003. Phylogeny of sheep and goat Theileria and Babesia parasites. Parasitology Research, 91, 398-406.

53. Seong G, Han YJ, Chae JB, Chae JS, Yu DH, Lee YS, Park J, Park BK, Yoo JG, Choi KS. 2015. Detection of Anaplasma sp. in Korean Native Goats (Capra aegagrus hircus) on Jeju Island, Korea. Korean Journal of Parasitology, 53, 765-769.

54. Shabana II, Alhadlag NM, Zaraket H. 2018. Diagnostic tools of caprine and ovine anaplasmosis: a direct comparative study. BMC Veterinary Research, 14, 165.

55. Shah SSA, Khan MI, Rahman HU. 2017. Epidemiological and hematological investigations of tick-borne diseases in small ruminants in Peshawar and Khyber Agency, Pakistan. Journal of Advances in Parasitology, 4, 15-22.

56. Sneath PHA, Sokal RR. 1973. Numerical taxonomy: the principles and practice of numerical classification. San Francisco: W H Freeman \& Co (Sd).
57. Tageldin MH, Al-Kitany Fadiya A, Al-Yahyae Sabra A, Al-I Ismaily SI. 2005. Theileriosis in sheep and goats in the Sultanate of Oman. Tropical Animal Health and Production, 37, 491-493.

58. Ullah N, Durrani AZ, Avais M, Ahmad N, Ullah S, Khan MS, Mehmood K, Khan MA, Haq I. 2018. Prevalence, risk factors and host biomarkers of ovine Theileriosis. Pakistan Journal of Zoology, 50, 1211-1216.

59. Yousefi A, Rahbari S, Shayan P, Sadeghi-dehkordi Z, Bahonar A. 2017. Molecular detection of Anaplasma marginale and Anaplasma ovis in sheep and goat in west highland pasture of Iran. Asian Pacific Journal of Tropical Biomedicine, 7, 455459.

60. Yu Z-Q, Song J-K, Zhang H-J, Liu T-L, Fan X-C, Zhao G-H. 2018. Molecular characterization of Theileria spp. in goats from Shaanxi Province, Northwestern China. Journal of Parasitology, 104, 726-731.

61. Zaeemi M, Haddadzadeh H, Khazraiinia P, Kazemi B, Bandehpour M. 2011. Identification of different Theileria species (Theileria lestoquardi, Theileria ovis, and Theileria annulata) in naturally infected sheep using nested PCR-RFLP. Parasitology Research, 108, 837-843.

62. Zhan L, Cao W-C, Jiang J-F, Zhang X-A, Wu X-M, Zhang W-Y, Liu W, Zuo S-Q, Cao Z-W, Yang H, Richardus JH, Habbema JD. 2010. Anaplasma phagocytophilum in livestock and small rodents. Veterinary Microbiology, 144, 405-408.

63. Zhang X, Liu Z, Yang J, Chen Z, Guan G, Ren Q, Liu A, Luo J, Yin H, Li Y. 2014. Multiplex PCR for diagnosis of Theileria uilenbergi, Theileria luwenshuni, and Theileria ovis in small ruminants. Parasitology Research, 113, 527-531.

64. Zhang J, Kelly P, Li J, Xu C, Wang C. 2015. Molecular detection of Theileria spp. in livestock on five Caribbean islands. BioMed Research International, 2015, 624728.

65. Zia-ur-Rehman, Khan MS, Avais M, Aleem M, Shabbir MZ, Khan JA. 2010. Prevalence of theileriosis in sheep in Okara District, Pakistan. Pakistan Journal of Zoology, 42, 639-643.

66. Zulfiqar S, Shahnawaz S, Ali M, Bhutta AM, Iqbal S, Hayat S, Qadir S, Latif M, Kiran N, Saeed A, Ali M, Iqbal F. 2012. Detection of Babesia bovis in blood samples and its effect on the hematological and serum biochemical profile in large ruminants from Southern Punjab. Asian Pacific Journal of Tropical Biomedicine, 2, 104-108.

Cite this article as: Niaz S, Ur Rahman Z, Ali I, Cossío-Bayúgar R, Amaro-Estrada I, Alanazi AD, Khattak I, Zeb J, Nasreen N \& Khan A. 2021. Molecular prevalence, characterization and associated risk factors of Anaplasma spp. and Theileria spp. in small ruminants in Northern Pakistan. Parasite 28, 3. 
An international open-access, peer-reviewed, online journal publishing high quality papers on all aspects of human and animal parasitology

Reviews, articles and short notes may be submitted. Fields include, but are not limited to: general, medical and veterinary parasitology; morphology, including ultrastructure; parasite systematics, including entomology, acarology, helminthology and protistology, and molecular analyses; molecular biology and biochemistry; immunology of parasitic diseases; host-parasite relationships; ecology and life history of parasites; epidemiology; therapeutics; new diagnostic tools.

All papers in Parasite are published in English. Manuscripts should have a broad interest and must not have been published or submitted elsewhere. No limit is imposed on the length of manuscripts.

Parasite (open-access) continues Parasite (print and online editions, 1994-2012) and Annales de Parasitologie Humaine et Comparée (1923-1993) and is the official journal of the Société Française de Parasitologie. 